\title{
CANARDS EXISTENCE IN THE HINDMARSH-ROSE MODEL
}

\author{
JEAN-MARC GINOUX ${ }^{1}$, JAUME LLIBRE ${ }^{2}$ AND KIYOYUKI TCHIZAWA ${ }^{3}$
}

\begin{abstract}
In two previous papers we have proposed a new method for proving the existence of "canard solutions" on one hand for three and four-dimensional singularly perturbed systems with only one fast variable and, on the other hand for four-dimensional singularly perturbed systems with two fast variables. The aim of this work is to extend this method which improves the classical ones used till now to the case of three-dimensional singularly perturbed systems with two fast variables. This method enables to state a unique generic condition for the existence of "canard solutions" for such three-dimensional singularly perturbed systems which is based on the stability of folded singularities (pseudo singular points in this case) of the normalized slow dynamics deduced from a well-known property of linear algebra. Applications of this method to a famous neuronal bursting model enables to show the existence of "canard solutions" in the Hindmarsh-Rose model.
\end{abstract}

\section{INTRODUCTION}

The concept of "canard solutions" for three-dimensional singularly perturbed systems with one slow variables and two fast has been introduced in the beginning of the eighties by Benoit and Lobry [2], Benoît [3]. Their existence has been proved by Benoît [3, p. 170] in the framework of "Non-Standard Analysis" according to a theorem which states that canard solutions exist in such systems provided that the pseudo singular point of the slow dynamics, i.e., of the reduced vector field is of saddle type. Nearly twenty years later, Szmolyan and Wechselberger [21] provided a "standard version" of Benoît's theorem [3]. Recently, Wechselberger [27] generalized this theorem for $n$-dimensional singularly perturbed systems with $k$ slow variables and $m$ fast (where $n=k+m$ ). The method they used require to implement a "desingularization procedure" which can be summarized as follows: first, they compute the normal form of such singularly perturbed systems which is expressed according to some coefficients ( $a$ and $b$ for dimension three and $\tilde{a}, \tilde{b}$ and $\tilde{c}_{1}$ for dimension four) depending on the functions defining the original vector field and their partial derivatives with respect to the variables. Secondly, they project the "desingularized vector field" (originally called "normalized slow dynamics" by Eric Benoit [3, p. 166]) of such a normal form on the tangent bundle of the critical manifold. Finally, they evaluate the Jacobian of the projection of this "desingularized vector field" at the folded singularity (originally called pseudo singular points by José Argémi [1, p. 336]). This lead Szmolyan and Wechselberger $[21$, p. 427$]$ and Wechselberger $[27$, p. 3298] to a "classification of folded singularities (pseudo singular points)". Thus, they showed that for three-dimensional (resp.

2010 Mathematics Subject Classification. Primary 34C23, 34C25, 37G10.

Key words and phrases. Hindmarsh-Rose model; singularly perturbed dynamical systems; canard solutions. 
four-dimensional) singularly perturbed systems such folded singularity is of saddle type if the following condition is satisfied: $a<0$ (resp. $\tilde{a}<0$ ).

In a first paper entitled: "Canards Existence in Memristor's Circuits" (see Ginoux \& Llibre [11]) we presented a method enabling to state a unique "generic" condition for the existence of "canard solutions" for three and four-dimensional singularly perturbed systems with only one fast variable which is based on the stability of folded singularities of the normalized slow dynamics deduced from a well-known property of linear algebra. We proved that this unique condition is completely identical to that provided by Benoit [3], Szmolyan and Wechselberger [21] and Wechselberger [27].

In a second paper entitled: "Canards Existence in FitzHugh-Nagumo and HodgkinHuxley Neuronal Models" (see Ginoux \& Llibre [12]) we extended this method to the case of four-dimensional singularly perturbed systems with $k=2$ slow and $m=2$ fast variables. Then, we stated that the provided condition for the existence of canards is "generic" since it is exactly the same for singularly perturbed systems of dimension three and four with one or two fast variables. The method we used led us to the following proposition: If the normalized slow dynamics has a pseudo singular point of saddle type, i.e. if the sum $\sigma_{2}$ of all second-order diagonal minors of the Jacobian matrix of the normalized slow dynamics evaluated at the pseudo singular point is negative, i.e. if $\sigma_{2}<0$ then, the three-dimensional (resp. fourdimensional) singularly perturbed system exhibits a canard solution which evolves from the attractive part of the slow manifold towards its repelling part. Then, we proved on one hand for three-dimensional singularly perturbed systems with only one fast variable that the condition for which the pseudo singular point is of saddle type, i.e. $\sigma_{2}<0$ is identical to that proposed by Benoit [3, p. 171] in his theorem, i.e. $D<0$ and also to that provided by Szmolyan and Wechselberger [21], i.e. $a<0$. On the other hand, we proved for four-dimensional singularly perturbed systems with one or two fast variables that the condition for which the folded singularity (resp. the pseudo singular point) is of saddle type, i.e. $\sigma_{2}<0$ is identical to that proposed by Wechselberger [27, p. 3298] in his theorem, i.e. $\tilde{a}<0$. Such three and four-dimensional singularly perturbed systems with $k$ slow and $m=2$ fast variables have been also extensively studied by K. Tchizawa (see [24, 25]).

The aim of this work is to extend this method to the case of three-dimensional singularly perturbed systems with one slow and two fast variables and to show that the provided condition for the existence of canards, i.e. $\sigma_{2}<0$ still holds and is consequently "generic".

The outline of this paper is as follows. In Sec. 2, definitions of singularly perturbed system, critical manifold, reduced system, "constrained system", canard cycles, folded singularities and pseudo singular points are recalled. The method proposed in this article is presented in Sec. 3 for the case of three-dimensional singularly perturbed systems with two fast variables. In Sec. 4, applications of this method to the famous Hindmarsh-Rose model enables to show the existence of "canard solutions" in such system. 


\section{Definitions}

2.1. Singularly perturbed systems. According to Tikhonov [23], Jones [16] and Kaper [17] singularly perturbed systems are defined as:

$$
\begin{aligned}
\vec{x}^{\prime} & =\varepsilon \vec{f}(\vec{x}, \vec{y}, \varepsilon), \\
\vec{y}^{\prime} & =\vec{g}(\vec{x}, \vec{y}, \varepsilon),
\end{aligned}
$$

where $\vec{x} \in \mathbb{R}^{k}, \vec{y} \in \mathbb{R}^{m}, \varepsilon \in \mathbb{R}^{+}$, and the prime denotes differentiation with respect to the independent variable $t^{\prime}$. The functions $\vec{f}$ and $\vec{g}$ are assumed to be $C^{\infty}$ functions ${ }^{1}$ of $\vec{x}, \vec{y}$ and $\varepsilon$ in $U \times I$, where $U$ is an open subset of $\mathbb{R}^{k} \times \mathbb{R}^{m}$ and $I$ is an open interval containing $\varepsilon=0$.

In the case when $0<\varepsilon \ll 1$, i.e. $\varepsilon$ is a small positive number, the variable $\vec{x}$ is called slow variable, and $\vec{y}$ is called fast variable. Using Landau's notation: $O\left(\varepsilon^{p}\right)$ represents a function $f$ of $u$ and $\varepsilon$ such that $f(u, \varepsilon) / \varepsilon^{p}$ is bounded for positive $\varepsilon$ going to zero, uniformly for $u$ in the given domain.

In general we consider that $\vec{x}$ evolves at an $O(\varepsilon)$ rate; while $\vec{y}$ evolves at an $O(1)$ slow rate. Reformulating system (1) in terms of the rescaled variable $t=\varepsilon t^{\prime}$, we obtain

$$
\begin{aligned}
\dot{\vec{x}} & =\vec{f}(\vec{x}, \vec{y}, \varepsilon), \\
\varepsilon \dot{\vec{y}} & =\vec{g}(\vec{x}, \vec{y}, \varepsilon) .
\end{aligned}
$$

The dot represents the derivative with respect to the new independent variable $t$. The independent variables $t^{\prime}$ and $t$ are referred to the fast and slow times, respectively, and (1) and (2) are called the fast and slow systems, respectively. These systems are equivalent whenever $\varepsilon \neq 0$, and they are labeled singular perturbation problems when $0<\varepsilon \ll 1$. The label "singular" stems in part from the discontinuous limiting behavior in system (1) as $\varepsilon \rightarrow 0$.

2.2. Reduced slow system. When $\varepsilon \rightarrow 0$ system (2) leads to a system of differentialalgebraic equations (D.A.E.) called reduced slow system whose dimension decreases from $k+m=n$ to $m$. Then, the slow variable $\vec{x} \in \mathbb{R}^{k}$ partially evolves in the submanifold

$$
M_{0}:=\{(\vec{x}, \vec{y}): \vec{g}(\vec{x}, \vec{y}, 0)=\overrightarrow{0}\} .
$$

called the critical manifold ${ }^{2}$. The reduced slow system is

$$
\begin{aligned}
& \dot{\vec{x}}=\vec{f}(\vec{x}, \vec{y}, \varepsilon), \\
& \overrightarrow{0}=\vec{g}(\vec{x}, \vec{y}, \varepsilon) .
\end{aligned}
$$

2.3. Slow Invariant Manifold. Such a normally hyperbolic invariant manifold (3) of the reduced slow system (4) persists as a locally invariant slow manifold of the full system (1) for $\varepsilon$ sufficiently small. This locally slow invariant manifold is $O(\varepsilon)$ close to the critical manifold.

When $D_{\vec{x}} \vec{f}$ is invertible, thanks to the Implicit Function Theorem, $M_{0}$ is given by the graph of a $C^{\infty}$ function $\vec{x}=\vec{G}_{0}(\vec{y})$ for $\vec{y} \in D$, where $D \subseteq \mathbb{R}^{k}$ is a compact,

\footnotetext{
${ }^{1}$ In certain applications these functions will be supposed to be $C^{r}, r \geqslant 1$.

${ }^{2}$ It represents the approximation of the slow invariant manifold, with an error of $O(\varepsilon)$.
} 
simply connected domain and the boundary of $\mathrm{D}$ is a $(k-1)$-dimensional $C^{\infty}$ submanifold ${ }^{3}$.

According to Fenichel $[4,7]$ theory if $0<\varepsilon \ll 1$ is sufficiently small, then there exists a function $\vec{G}(\vec{y}, \varepsilon)$ defined on D such that the manifold

$$
M_{\varepsilon}:=\{(\vec{x}, \vec{y}): \vec{x}=\vec{G}(\vec{y}, \varepsilon)\},
$$

is locally invariant under the flow of system (1). Moreover, there exist perturbed local stable (or attracting) $M_{a}$ and unstable (or repelling) $M_{r}$ branches of the slow invariant manifold $M_{\varepsilon}$. Thus, normal hyperbolicity of $M_{\varepsilon}$ is lost via a saddlenode bifurcation of the reduced slow system (4). Then, it gives rise to solutions of "canard" type.

2.4. Canards, singular canards and maximal canards. A canard is a solution of a singularly perturbed dynamical system (1) following the attracting branch $M_{a}$ of the slow invariant manifold, passing near a bifurcation point located on the fold of this slow invariant manifold, and then following the repelling branch $M_{r}$ of the slow invariant manifold.

A singular canard is a solution of a reduced slow system (4) following the attracting branch $M_{a, 0}$ of the critical manifold, passing near a bifurcation point located on the fold of this critical manifold, and then following the repelling branch $M_{r, 0}$ of the critical manifold.

A maximal canard corresponds to the intersection of the attracting and repelling branches $M_{a, \varepsilon} \cap M_{r, \varepsilon}$ of the slow manifold in the vicinity of a non-hyperbolic point.

According to Wechselberger [27, p. 3302]:

"Such a maximal canard defines a family of canards nearby which are exponentially close to the maximal canard, i.e. a family of solutions of (1) that follow an attracting branch $M_{a, \varepsilon}$ of the slow manifold and then follow, rather surprisingly, a repelling/saddle branch $M_{r, \varepsilon}$ of the slow manifold for a considerable amount of slow time. The existence of this family of canards is a consequence of the non-uniqueness of $M_{a, \varepsilon}$ and $M_{r, \varepsilon}$. However, in the singular limit $\varepsilon \rightarrow 0$, such a family of canards is represented by a unique singular canard."

Canards are a special class of solution of singularly perturbed dynamical systems for which normal hyperbolicity is lost. Canards in singularly perturbed systems with two or more slow variables $\left(\vec{x} \in \mathbb{R}^{k}, k \geqslant 2\right)$ and one fast variable $\left(\vec{y} \in \mathbb{R}^{m}\right.$, $m=1)$ are robust, since maximal canards generically persist under small parameter changes ${ }^{4}$.

2.5. Constrained system. In order to characterize the "slow dynamics", i.e. the slow trajectory of the reduced slow system (4) (obtained by setting $\varepsilon=0$ in (2)),

\footnotetext{
${ }^{3}$ The set $\mathrm{D}$ is overflowing invariant with respect to $(2)$ when $\varepsilon=0$. See Kaper [17] and Jones [16].

${ }^{4}$ See Benoît [3], Szmolyan and Wechselberger [21] and Wechselberger [26, 27].
} 
Floris Takens [22] introduced the "constrained system" defined as follows:

$$
\begin{aligned}
\dot{\vec{x}} & =\vec{f}(\vec{x}, \vec{y}, 0), \\
D_{\vec{y}} \vec{g} \cdot \dot{\vec{y}} & =-\left(D_{\vec{x}} \vec{g} \cdot \vec{f}\right)(\vec{x}, \vec{y}, 0), \\
\overrightarrow{0} & =\vec{g}(\vec{x}, \vec{y}, 0) .
\end{aligned}
$$

Since, according to Fenichel $[4,7]$, the critical manifold $\vec{g}(\vec{x}, \vec{y}, 0)$ may be considered as locally invariant under the flow of system (1), we have:

$$
\frac{d \vec{g}}{d t}(\vec{x}, \vec{y}, 0)=0 \quad \Longleftrightarrow \quad D_{\vec{x}} \vec{g} \cdot \dot{\vec{x}}+D_{\vec{y}} \vec{g} \cdot \dot{\vec{y}}=\overrightarrow{0} .
$$

By replacing $\dot{\vec{x}}$ by $\vec{f}(\vec{x}, \vec{y}, 0)$ leads to:

$$
D_{\vec{x}} \vec{g} \cdot \vec{f}(\vec{x}, \vec{y}, 0)+D_{\vec{y}} \vec{g} \cdot \dot{\vec{y}}=\overrightarrow{0} .
$$

This justifies the introduction of the constrained system.

Now, let $\operatorname{adj}\left(D_{\vec{y}} \vec{g}\right)$ denote the adjoint of the matrix $D_{\vec{y}} \vec{g}$ which is the transpose of the co-factor matrix $D_{\vec{y}} \vec{g}$, then while multiplying the left hand side of (6) by the inverse matrix $\left(D_{\vec{y}} \vec{g}\right)^{-1}$ obtained by the adjoint method we have:

$$
\begin{aligned}
\dot{\vec{x}} & =\vec{f}(\vec{x}, \vec{y}, 0), \\
\operatorname{det}\left(D_{\vec{y}} \vec{g}\right) \dot{\vec{y}} & =-\left(\operatorname{adj}\left(D_{\vec{y}} \vec{g}\right) \cdot D_{\vec{x}} \vec{g} \cdot \vec{f}\right)(\vec{x}, \vec{y}, 0), \\
\overrightarrow{0} & =\vec{g}(\vec{x}, \vec{y}, 0) .
\end{aligned}
$$

2.6. Normalized slow dynamics. By rescaling the time by setting $t=-\operatorname{det}\left(D_{\vec{y}} \vec{g}\right) \tau$ in system (7) we obtain the following system which has been called by Eric Benoît [3, p. 166] "normalized slow dynamics":

$$
\begin{aligned}
\dot{\vec{x}} & =-\operatorname{det}\left(D_{\vec{y}} \vec{g}\right) \vec{f}(\vec{x}, \vec{y}, 0), \\
\dot{\vec{y}} & =\left(\operatorname{adj}\left(D_{\vec{y}} \vec{g}\right) \cdot D_{\vec{x}} \vec{g} \cdot \vec{f}\right)(\vec{x}, \vec{y}, 0), \\
\overrightarrow{0} & =\vec{g}(\vec{x}, \vec{y}, 0) .
\end{aligned}
$$

where the overdot now denotes the time derivation with respect to $\tau$. We notice that Argémi [1] proposed to rescale the time by setting $t=-\operatorname{det}\left(D_{\vec{y}} \vec{g}\right) \operatorname{sgn}\left(\operatorname{det}\left(D_{\vec{y}} \vec{g}\right)\right) \tau$ in order to keep the same flow direction in (8) as in (7).

2.7. Desingularized vector field. By application of the Implicit Function Theorem, we suppose that we can explicitly express from Eq. (3), say without loss of generality, $x_{1}$ as a function $\phi_{1}$ of the other variables. This implies that $M_{0}$ is locally the graph of a function $\phi_{1}: \mathbb{R}^{k} \rightarrow \mathbb{R}^{m}$ over the base $U=(\vec{\chi}, \vec{y})$ where $\vec{\chi}=\left(x_{2}, x_{3}, \ldots, x_{k}\right)$. Thus, we can span the "normalized slow dynamics" on the tangent bundle at the critical manifold $M_{0}$ at the pseudo singular point. This leads to the so-called desingularized vector field:

$$
\begin{aligned}
& \dot{\vec{\chi}}=-\operatorname{det}\left(D_{\vec{y}} \vec{g}\right) \vec{f}(\vec{\chi}, \vec{y}, 0), \\
& \dot{\vec{y}}=\left(\operatorname{adj}\left(D_{\vec{y}} \vec{g}\right) \cdot D_{\vec{x}} \vec{g} \cdot \vec{f}\right)(\vec{\chi}, \vec{y}, 0) .
\end{aligned}
$$


2.8. Pseudo singular points and folded singularities. As recalled by Guckenheimer and Haiduc [13, p. 91], pseudo-singular points have been introduced by the late José Argémi [1] for low-dimensional singularly perturbed systems and are defined as singular points of the "normalized slow dynamics" (8). Twenty-three years later, Szmolyan and Wechselberger [21, p. 428] called such pseudo singular points, folded singularities. In a recent publication entitled "A propos de canards" Wechselberger [27, p. 3295] proposed to define such singularities for $n$-dimensional singularly perturbed systems with $k$ slow variables and $m$ fast as the solutions of the following system:

$$
\begin{aligned}
& \operatorname{det}\left(D_{\vec{y}} \vec{g}\right)=0, \\
& \left(\operatorname{adj}\left(D_{\vec{y}} \vec{g}\right) \cdot D_{\vec{x}} \vec{g} \cdot \vec{f}\right)(\vec{x}, \vec{y}, 0)=\overrightarrow{0}, \\
& \vec{g}(\vec{x}, \vec{y}, 0)=\overrightarrow{0} .
\end{aligned}
$$

Thus, for dimensions higher than three, his concept encompasses that of Argémi. Moreover, for $k \geqslant 2$ ), Wechselberger [27, p. 3296] proved that folded singularities form a $(k-2)$-dimensional manifold. Thus, for $k=2$ the folded singularities are nothing else than the pseudo singular points defined by Argémi [1]. Nevertheless, for the degenerate case $k=1$, folded singularities still form a zero-dimensional manifold. So, we will see in the next Sec. 3 that the stability analysis of the pseudo singular points will give rise to a condition for the existence of canard solutions in the original system (1).

\section{Three-Dimensional Singularly PERTURBED Systems With TWO FAST VARIABLES}

A three-dimensional singularly perturbed dynamical system (2) with $k=1$ slow variables and $m=2$ fast may be written as:

$$
\begin{aligned}
\dot{x}_{1} & =f_{1}\left(x_{1}, y_{1}, y_{2}\right), \\
\varepsilon \dot{y}_{1} & =g_{1}\left(x_{1}, y_{1}, y_{2}\right), \\
\varepsilon \dot{y}_{2} & =g_{2}\left(x_{1}, y_{1}, y_{2}\right),
\end{aligned}
$$

where $x_{1} \in \mathbb{R}, \vec{y}=\left(y_{1}, y_{2}\right)^{t} \in \mathbb{R}^{2}, 0<\varepsilon \ll 1$ and the functions $f_{i}$ and $g_{i}$ are assumed to be $C^{2}$ functions of $\left(x_{1}, y_{1}, y_{2}\right)$.

3.1. Critical Manifold. The critical manifold equation of system (11) is defined by setting $\varepsilon=0$ in Eqs. (11b \& 11c). Thus, we obtain:

$$
\begin{aligned}
& g_{1}\left(x_{1}, y_{1}, y_{2}\right)=0, \\
& g_{2}\left(x_{1}, y_{1}, y_{2}\right)=0 .
\end{aligned}
$$

By application of the Implicit Function Theorem, we suppose that we can explicitly express from Eqs. (12a \& 12b), say without loss of generality, $x_{1}$ and $y_{1}$ as functions of the others variables:

$$
\begin{aligned}
& y_{1}=\phi_{1}\left(x_{1}, y_{2}\right), \\
& x_{1}=\phi_{2}\left(y_{1}, y_{2}\right) .
\end{aligned}
$$


3.2. Constrained system. The constrained system is obtained by equating to zero the time derivative of $g_{1,2}\left(x_{1}, y_{1}, y_{2}\right)$ :

$$
\begin{aligned}
\frac{d g_{1}}{d t} & =\frac{\partial g_{1}}{\partial x_{1}} \dot{x}_{1}+\frac{\partial g_{1}}{\partial y_{1}} \dot{y}_{1}+\frac{\partial g_{1}}{\partial y_{2}} \dot{y}_{2}=0 \\
\frac{d g_{2}}{d t} & =\frac{\partial g_{2}}{\partial x_{1}} \dot{x}_{1}+\frac{\partial g_{2}}{\partial y_{1}} \dot{y}_{1}+\frac{\partial g_{2}}{\partial y_{2}} \dot{y}_{2}=0
\end{aligned}
$$

Eqs. (14a \& 14b) may be written as:

$$
\begin{aligned}
& \frac{\partial g_{1}}{\partial y_{1}} \dot{y}_{1}+\frac{\partial g_{1}}{\partial y_{2}} \dot{y}_{2}=-\frac{\partial g_{1}}{\partial x_{1}} \dot{x}_{1} \\
& \frac{\partial g_{2}}{\partial y_{1}} \dot{y}_{1}+\frac{\partial g_{2}}{\partial y_{2}} \dot{y}_{2}=-\frac{\partial g_{2}}{\partial x_{1}} \dot{x}_{1}
\end{aligned}
$$

By solving the system of two equations (15a \& 15b) with two unknowns $\left(\dot{y}_{1}, \dot{y}_{2}\right)$ we find:

$$
\begin{gathered}
\dot{y}_{1}=-\frac{\left(\frac{\partial g_{1}}{\partial x_{1}} \frac{\partial g_{2}}{\partial y_{2}}-\frac{\partial g_{1}}{\partial y_{2}} \frac{\partial g_{2}}{\partial x_{1}}\right) \dot{x}_{1}}{\operatorname{det}\left[J_{\left(y_{1}, y_{2}\right)}\right]}, \\
\dot{y}_{2}=-\frac{\left(\frac{\partial g_{1}}{\partial y_{1}} \frac{\partial g_{2}}{\partial x_{1}}-\frac{\partial g_{1}}{\partial x_{1}} \frac{\partial g_{2}}{\partial y_{1}}\right) \dot{x}_{1}}{\operatorname{det}\left[J_{\left(y_{1}, y_{2}\right)}\right]} .
\end{gathered}
$$

So, we have the following constrained system:

$$
\begin{aligned}
\dot{x}_{1} & =f_{1}\left(x_{1}, y_{1}, y_{2}\right), \\
\dot{y}_{1} & =-\frac{\left(\frac{\partial g_{1}}{\partial x_{1}} \frac{\partial g_{2}}{\partial y_{2}}-\frac{\partial g_{1}}{\partial y_{2}} \frac{\partial g_{2}}{\partial x_{1}}\right) \dot{x}_{1}}{\operatorname{det}\left[J_{\left(y_{1}, y_{2}\right)}\right]}, \\
\dot{y}_{2} & =-\frac{\left(\frac{\partial g_{1}}{\partial y_{1}} \frac{\partial g_{2}}{\partial x_{1}}-\frac{\partial g_{1}}{\partial x_{1}} \frac{\partial g_{2}}{\partial y_{1}}\right) \dot{x}_{1}}{\operatorname{det}\left[J_{\left(y_{1}, y_{2}\right)}\right]}, \\
0 & =g_{1}\left(x_{1}, y_{1}, y_{2}\right), \\
0 & =g_{2}\left(x_{1}, y_{1}, y_{2}\right) .
\end{aligned}
$$

3.3. Normalized slow dynamics. By rescaling the time by setting $t=-\operatorname{det}\left[J_{\left(y_{1}, y_{2}\right)}\right] \tau$ we obtain the "normalized slow dynamics":

$$
\begin{aligned}
\dot{x}_{1} & =-f_{1}\left(x_{1}, y_{1}, y_{2}\right) \operatorname{det}\left[J_{\left(y_{1}, y_{2}\right)}\right]=F_{1}\left(x_{1}, y_{1}, y_{2}\right), \\
\dot{y}_{1} & =f_{1}\left(x_{1}, y_{1}, y_{2}\right)\left(\frac{\partial g_{1}}{\partial x_{1}} \frac{\partial g_{2}}{\partial y_{2}}-\frac{\partial g_{1}}{\partial y_{2}} \frac{\partial g_{2}}{\partial x_{1}}\right)=G_{1}\left(x_{1}, y_{1}, y_{2}\right), \\
\dot{y}_{2} & =f_{1}\left(x_{1}, y_{1}, y_{2}\right)\left(\frac{\partial g_{1}}{\partial y_{1}} \frac{\partial g_{2}}{\partial x_{1}}-\frac{\partial g_{1}}{\partial x_{1}} \frac{\partial g_{2}}{\partial y_{1}}\right)=G_{2}\left(x_{1}, y_{1}, y_{2}\right), \\
0 & =g_{1}\left(x_{1}, y_{1}, y_{2}\right), \\
0 & =g_{2}\left(x_{1}, y_{1}, y_{2}\right),
\end{aligned}
$$

where the overdot now denotes the time derivation with respect to $\tau$. 
3.4. Desingularized system on the critical manifold. Since we have supposed that $y_{1}$ and $y_{2}$ may be explicitly expressed as functions of the others variables (13a $\& 13 \mathrm{~b}$ ), they can be used to project the normalized slow dynamics (18) on the tangent bundle of the critical manifold. So, we have:

$$
\begin{aligned}
& \dot{x}_{1}=-f_{1}\left(x_{1}, y_{1}, y_{2}\right) \operatorname{det}\left[J_{\left(y_{1}, y_{2}\right)}\right]=F_{1}\left(x_{1}, y_{2}\right), \\
& \dot{y}_{2}=f_{1}\left(x_{1}, y_{1}, y_{2}\right)\left(\frac{\partial g_{1}}{\partial y_{1}} \frac{\partial g_{2}}{\partial x_{1}}-\frac{\partial g_{1}}{\partial x_{1}} \frac{\partial g_{2}}{\partial y_{1}}\right)=G_{2}\left(x_{1}, y_{2}\right) .
\end{aligned}
$$

3.5. Pseudo singular points. Pseudo-singular points are defined as singular points of the "normalized slow dynamics", i.e. as the set of points for which we have:

$$
\begin{aligned}
& \operatorname{det}\left[J_{\left(y_{1}, y_{2}\right)}\right]=0, \\
& \left(\frac{\partial g_{1}}{\partial x_{1}} \frac{\partial g_{2}}{\partial y_{2}}-\frac{\partial g_{1}}{\partial y_{2}} \frac{\partial g_{2}}{\partial x_{1}}\right)=0, \\
& \left(\frac{\partial g_{1}}{\partial y_{1}} \frac{\partial g_{2}}{\partial x_{1}}-\frac{\partial g_{1}}{\partial x_{1}} \frac{\partial g_{2}}{\partial y_{1}}\right)=0, \\
& g_{1}\left(x_{1}, y_{1}, y_{2}\right)=0, \\
& g_{2}\left(x_{1}, y_{1}, y_{2}\right)=0 .
\end{aligned}
$$

Remark. We notice on the one hand that Eqs. (20b) \& (20c) are linearly dependent and on the other hand that contrary to previous works we do not use the "desingularized vector field" (19) but the "normalized slow dynamics" (18).

The Jacobian matrix of system (18) reads:

$$
J_{\left(F_{1}, G_{1}, G_{2}\right)}=\left(\begin{array}{lll}
\frac{\partial F_{1}}{\partial x_{1}} & \frac{\partial F_{1}}{\partial y_{1}} & \frac{\partial F_{1}}{\partial y_{2}} \\
\frac{\partial G_{1}}{\partial x_{1}} & \frac{\partial G_{1}}{\partial y_{1}} & \frac{\partial G_{1}}{\partial y_{2}} \\
\frac{\partial G_{2}}{\partial x_{1}} & \frac{\partial G_{2}}{\partial y_{1}} & \frac{\partial G_{2}}{\partial y_{2}}
\end{array}\right) .
$$

3.6. Extension of Benoît's generic hypothesis. Without loss of generality, it seems reasonable to extend Benoit's generic hypotheses introduced for threedimensional singularly perturbed systems with only one fast variable to the case of two variables. So, first, we suppose that by a "standard translation" the pseudo singular point can be shifted at the origin $O(0,0,0)$ and that by a "standard rotation" of $y_{1}$-axis that the slow manifold is tangent to $\left(x_{1}, y_{1}\right)$-plane, so we have

$$
\begin{aligned}
& g_{1}(0,0,0)=g_{2}(0,0,0)=0, \\
& \left.\frac{\partial g_{1}}{\partial y_{1}}\right|_{(0,0,0)}=\left.\frac{\partial g_{2}}{\partial y_{1}}\right|_{(0,0,0)}=0, \\
& \left.\frac{\partial g_{1}}{\partial y_{2}}\right|_{(0,0,0)}=\left.\frac{\partial g_{2}}{\partial y_{2}}\right|_{(0,0,0)}=0 .
\end{aligned}
$$

Then, we make the following assumptions for the non-degeneracy of the pseudo singular point:

$$
f_{1}(0,0,0) \neq 0 .
$$


Thus, we have the following Cayley-Hamilton eigenpolynomial associated with such a Jacobian matrix (21) evaluated at the pseudo singular point, i.e. at the origin:

$$
\lambda^{3}-\sigma_{1} \lambda^{2}+\sigma_{2} \lambda-\sigma_{3}=0 .
$$

First, it can be proved that the sum of all first-order diagonal minors of $J_{\left(F_{1}, G_{1}, G_{2}\right)}$, i.e. $\sigma_{1}=\operatorname{Tr}\left(J_{\left(F_{1}, G_{1}, G_{2}\right)}\right)=0$ due to circular permutations of the partial derivatives. Secondly, it can also be proved that $\sigma_{3}=\left|J_{\left(F_{1}, G_{1}, G_{2}\right)}\right|=0$ vanishes at a pseudo singular point provided that generic condition $(22 \mathrm{~b})$ is satisfied. So, the eigenpolynomial (24) is reduced to

$$
\lambda\left(\lambda^{2}+\sigma_{2}\right)=0
$$

Let $\lambda_{i}$ be the eigenvalues of the eigenpolynomial (25) and we denote by $\lambda_{3}=0$ the obvious root of this polynomial. We have:

$$
\sigma_{2}=\sum_{i=1}^{3}\left|J_{\left(F_{1}, G_{1}, G_{2}\right)}^{i i}\right|=\lambda_{1} \lambda_{2} .
$$

where $\sigma_{2}=\sum_{i=1}^{3}\left|J_{\left(F_{1}, G_{1}, G_{2}\right)}^{i i}\right|=q$ represents the sum of all second-order diagonal minors of $J_{\left(F_{1}, G_{1}, G_{2}\right)}$. Obviously, the pseudo singular point is of saddle-type if and only if $\sigma_{2}<0$. This leads to the the following condition:

$$
C_{1}: \quad q<0 .
$$

3.7. Canard existence in $\mathbb{R}^{1+2}$. In an article entitled "Systèmes lents-rapides dans $\mathbb{R}^{3}$ et leurs canards", Benoit [3, p. 171] has stated in the framework of "nonstandard analysis" a theorem that can be written as follows:

Benoît's theorem [1983]. If the desingularized vector field (17) has a pseudo singular point of saddle type, then system (11) exhibits a canard solution which evolves from the attractive part of the slow manifold towards its repelling part. A few years later, Szmolyan and Wechselberger [21] gave a "standard version" of Benoit's theorem [3] (see Benoit's theorem above) for three-dimensional singularly perturbed systems with $k=2$ slow variables and $m=1$ fast. While using "standard analysis" and blow-up technique, Szmolyan and Wechselberger [21, p. 427] stated in their Lemma 2.1, while using "a smooth change of coordinates" (see Ginoux et al. $[11,12])$, that the original system can be transformed into a "normal form" (28) from which they deduced that the condition for the pseudo singular point to be of saddle type is $a<0$. Then, they proved the existence of canard solutions for the original system according to their Theorem 4.1(a).

In our previous papers (see Ginoux et al. $[11,12]$ ), we have established the following Prop. 1 for three and four-dimensional singularly perturbed systems with $k=1$ fast variable and for four-dimensional singularly perturbed systems with $k=2$ fast variables. In this work we show that this proposition still holds for three-dimensional singularly perturbed systems with $k=2$ fast variables and $m=1$ slow variable.

Proposition 1. If the normalized slow dynamics (18) has a pseudo singular point of saddle type, i.e. if the sum $\sigma_{2}$ of all second-order diagonal minors of the Jacobian matrix of the normalized slow dynamics (18) evaluated at the pseudo singular point is negative, i.e. if $\sigma_{2}<0$ then, system (11) exhibits a canard solution which evolves from the attractive part of the slow manifold towards its repelling part. 
Proof. The method used by Benoît [3], Szmolyan and Wechselberger [21] and Wechselberger [27] requires to implement a "desingularization procedure" which implies to project the "normalized slow dynamics" (18) on the tangent bundle of the critical manifold and, to evaluate the Jacobian of the projection of this "desingularized system" (19) at the pseudo singular points. As previously recalled, the method presented in this paper does not use the "desingularized system" (18) but the "normalized slow dynamics" (19). So, to prove the Prop. 1, we have just to show that the determinant of the Jacobian of the "desingularized system" (18) is identical to the sum $\sigma_{2}$ of all second-order diagonal minors of the Jacobian matrix of the "normalized slow dynamics" (19). According to Eq. (26):

$$
\sigma_{2}=\sum_{i=1}^{3}\left|J_{\left(F_{1}, G_{1}, G_{2}\right)}^{i i}\right|=\left|J_{\left(F_{1}, G_{1}, G_{2}\right)}^{11}\right|+\left|J_{\left(F_{1}, G_{1}, G_{2}\right)}^{22}\right|+\left|J_{\left(F_{1}, G_{1}, G_{2}\right)}^{33}\right| .
$$

While using the generic hypotheses Eqs. (22), it is easy to prove that:

$$
\left|J_{\left(F_{1}, G_{1}, G_{2}\right)}^{11}\right|=\left|J_{\left(F_{1}, G_{1}, G_{2}\right)}^{33}\right|=0
$$

The remaining determinant $\left|J_{\left(F_{1}, G_{1}, G_{2}\right)}^{22}\right|$ is exactly that of the "desingularized system" (19). So, Prop. 1 can be also used to state the existence of canard solution for such systems.

\section{Canards Existence in the Hindmarsh-Rose model}

The Hindmarsh-Rose model [14] describes the basic properties of individual neurons and appears as a reduction of the conductance based in the Hodgkin-Huxley model for neural spiking, see for more details [15]. Thus, the three-dimensional Hindmarsh-Rose polynomial ordinary differential system was originally written as:

$$
\begin{aligned}
& \frac{d x}{d t}=y-a x^{3}+b x^{2}-z+I, \\
& \frac{d y}{d t}=c-d x^{2}-y, \\
& \frac{d z}{d t}=r[s(x-\alpha)-z],
\end{aligned}
$$

where $x$ is a transmembrane neuron potential, $y$ and $z$ are the characteristics of ionic currents dynamic, $I$ is ambient current. The other parameters $(a, b, c, d$, $I, s, \alpha$ and $r$ ) reflect the physical features of the neurons and the dot indicates derivative with respect to the time $t$. We notice that the parameter $r<<1$. Existence of canard solutions in such system (28) has been originally suspected by Shilnikov et al. [19, p. 2149] and highlighted by Shchepakina [20]. Thus, according to the previous definitions, the Hindmarsh-Rose model may be written as a threedimensional singularly perturbed system with $k=1$ slow variable and $m=2$ fast variables. By posing $x \rightarrow y_{2}, y \rightarrow y_{1}, z \rightarrow x_{1}$ and $t^{\prime} \rightarrow \varepsilon t$ with $\varepsilon=r$, we obtain:

$$
\begin{aligned}
\dot{x}_{1} & =f_{1}\left(x_{1}, y_{1}, y_{2}\right)=s\left(y_{2}-\alpha\right)-x_{1}, \\
\varepsilon \dot{y}_{1} & =g_{1}\left(x_{1}, y_{1}, y_{2}\right)=c-d y_{2}^{2}-y_{1}, \\
\varepsilon \dot{y}_{2} & =g_{2}\left(x_{1}, y_{1}, y_{2}\right)=y_{1}-a y_{2}^{3}+b y_{2}^{2}-x_{1}+I,
\end{aligned}
$$


where $x_{1} \in \mathbb{R}, \vec{y}=\left(y_{1}, y_{2}\right)^{t} \in \mathbb{R}^{2}, 0<\varepsilon \ll 1$ and the functions $f_{i}$ and $g_{i}$ are assumed to be $C^{2}$ functions of $\left(x_{1}, y_{1}, y_{2}\right)$ and the dot now indicates derivative with respect to the time $t^{\prime}$.

4.1. Critical Manifold. The critical manifold equation of system (29) is defined by setting $\varepsilon=0$ in Eqs. (29b \& 29c). Thus, we have:

$$
\begin{aligned}
& g_{1}\left(x_{1}, y_{1}, y_{2}\right)=c-d y_{2}^{2}-y_{1}=0, \\
& g_{2}\left(x_{1}, y_{1}, y_{2}\right)=y_{1}-a y_{2}^{3}+b y_{2}^{2}-x_{1}+I=0 .
\end{aligned}
$$

This leads to the following critical manifold equation:

$$
y_{1}=\phi\left(y_{2}\right)=c+I-a y_{2}^{3}+(b-d) y_{2}^{2} .
$$

4.2. Constrained system. According to Eqs. (17), we have the following constrained system:

$$
\begin{aligned}
\dot{x}_{1} & =f_{1}\left(x_{1}, y_{1}, y_{2}\right)=s\left(y_{2}-\alpha\right)-x_{1}, \\
\dot{y}_{1} & =-\frac{-2 d y_{2} f_{1}\left(x_{1}, y_{1}, y_{2}\right)}{3 a y_{2}^{2}-2(b-d) y_{2}}, \\
\dot{y}_{2} & =-\frac{f_{1}\left(x_{1}, y_{1}, y_{2}\right)}{3 a y_{2}^{2}-2(b-d) y_{2}}, \\
0 & =c-d y_{2}^{2}-y_{1}, \\
0 & =y_{1}-a y_{2}^{3}+b y_{2}^{2}-x_{1}+I .
\end{aligned}
$$

4.3. Normalized slow dynamics. By rescaling the time by setting $t^{\prime}=-\left(3 a y_{2}^{2}\right.$ $\left.-2(b-d) y_{2}\right) \tau$ we obtain the "normalized slow dynamics":

$$
\begin{aligned}
\dot{x}_{1} & =-\left[3 a y_{2}^{2}-2(b-d) y_{2}\right]\left[s\left(y_{2}-\alpha\right)-x_{1}\right]=F_{1}\left(x_{1}, y_{1}, y_{2}\right), \\
\dot{y}_{1} & =-2 d y_{2}\left[s\left(y_{2}-\alpha\right)-x_{1}\right]=G_{1}\left(x_{1}, y_{1}, y_{2}\right), \\
\dot{y}_{2} & =\left[s\left(y_{2}-\alpha\right)-x_{1}\right]=G_{2}\left(x_{1}, y_{1}, y_{2}\right), \\
0 & =c-d y_{2}^{2}-y_{1}, \\
0 & =y_{1}-a y_{2}^{3}+b y_{2}^{2}-x_{1}+I .
\end{aligned}
$$

4.4. Desingularized system on the critical manifold. The projection of the normalized slow dynamics (32) on the tangent bundle of the critical manifold reads:

$$
\begin{aligned}
& \dot{x}_{1}=-\left[3 a y_{2}^{2}-2(b-d) y_{2}\right]\left[s\left(y_{2}-\alpha\right)-x_{1}\right]=F_{1}\left(x_{1}, y_{2}\right), \\
& \dot{y}_{2}=\left[s\left(y_{2}-\alpha\right)-x_{1}\right]=G_{2}\left(x_{1}, y_{2}\right) .
\end{aligned}
$$

4.5. Pseudo singular points. According to Eqs. (20) the pseudo-singular points of system (29) are:

$$
\begin{aligned}
& \left(\tilde{x}_{1}, \tilde{y}_{1}, \tilde{y}_{2}\right)=(c+I, c, 0), \\
& \left(\tilde{x}_{1}, \tilde{y}_{1}, \tilde{y}_{2}\right)=\left(\tilde{y}_{1}-a \tilde{y}_{2}^{3}+b \tilde{y}_{2}^{2}+I, c-d \tilde{y}_{2}^{2}, \frac{2}{3 a}(b-d)\right) .
\end{aligned}
$$


4.6. Canard existence. The Jacobian matrix of system (33) evaluated at the pseudo singular points (35a) reads:

$$
J_{\left(F_{1}, G_{1}, G_{2}\right)}=\left(\begin{array}{ccc}
0 & 0 & -2(b-d)(c+I+s \alpha) \\
0 & 0 & 2 d(c+I+s \alpha) \\
-1 & 0 & s
\end{array}\right) .
$$

According to Eqs. (26) we find that:

$$
\begin{aligned}
& p=\sigma_{1}=\operatorname{Tr}[J]=s, \\
& q=\sigma_{2}=-2(b-d)(c+I+s \alpha) .
\end{aligned}
$$

Thus, according to Prop. 1, the pseudo singular point is of saddle-type if:

$$
-2(b-d)(c+I+s \alpha)<0 .
$$

In her work Shchepakina [20] used the following parameter set: $a=1, b=3$, $c=1, d=0.275255, I=2.7$ and $\alpha=-1.2$. She found a canard without head (see Fig. 1) for the "duck parameter" value $s=3.0810445478558141214$. According to Eq. (37) and with such a parameter set, i.e. $b-d>0$, the pseudo singular point is of saddle-type if and only if:

$$
s<\frac{c+I}{\alpha} .
$$

With $c=1, I=2.7$ and $\alpha=-1.2$, we find that: $s<3.0833$. Thus, Shchepakina highlighted a canard without head in the Hindmarsh-Rose model (see Fig. 1) for the "duck parameter" value $s=3.0810445478558141214<3.0833$.

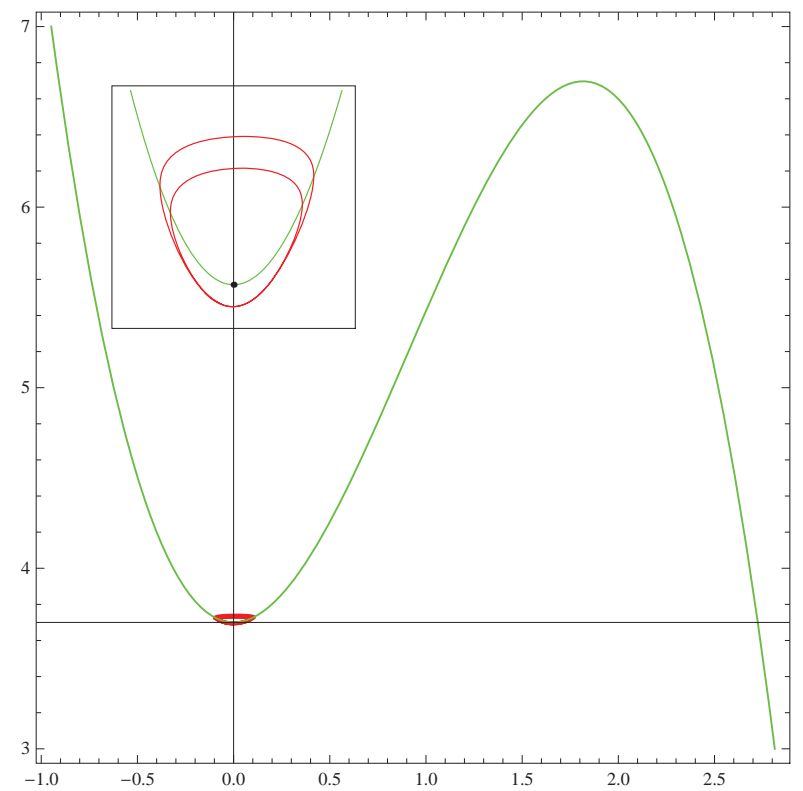

FiguRe 1. Canard solution of the Hindmarsh-Rose (28) model in the $(x, z)$ plane phase with the following parameter set: $a=1$, $b=3, c=1, d=0.275255, I=2.7, \alpha=-1.2$ and for the "duck parameter" value $s=3.0810445478558141214$. 
In the inset of Fig. 1, the zoom in highlights a large distance between the canard solution and that of the critical manifold (31). This is due to the fact that this latter corresponds to zero-order approximation in $\varepsilon$ of the slow invariant manifold. Nevertheless, while using the so-called Flow Curvature Method Ginoux and Rossetto [9] have already provided a second-order approximation in $\varepsilon$ of the slow invariant manifold of the Hindmarsh-Rose model (28). The result is presented in Fig. 2.

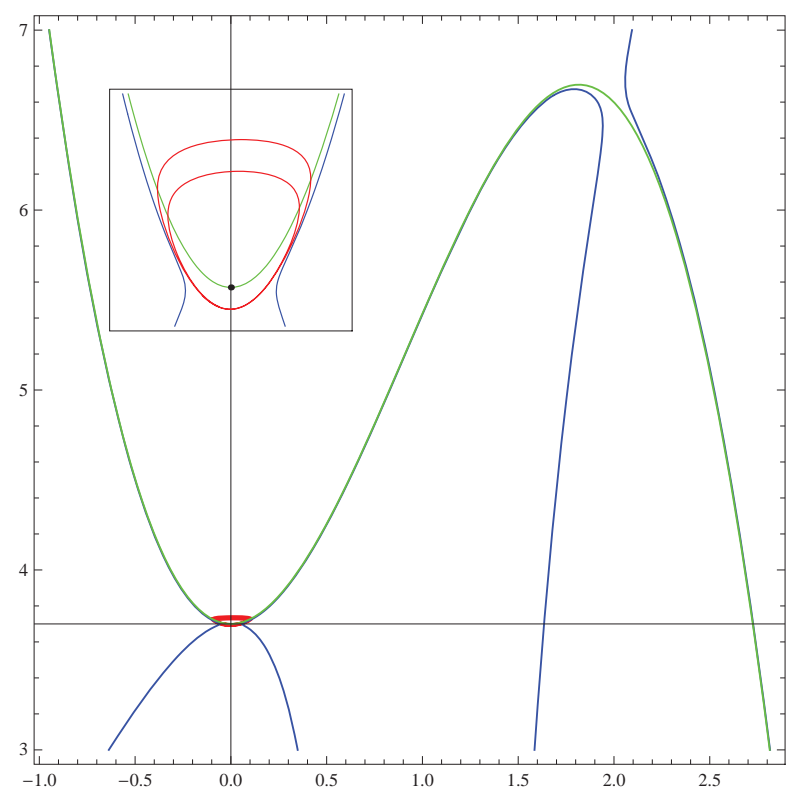

Figure 2. Canard solution of the Hindmarsh-Rose (28) model in the $(x, z)$ plane phase, its critical manifold (in green) and the second-order approximation in $\varepsilon$ of the slow invariant manifold (in blue) with the following parameter set: $a=1, b=3, c=1$, $d=0.275255, I=2.7, \alpha=-1.2$ and for the "duck parameter" value $s=3.0810445478558141214$.

Remark. We notice on the one hand that the Flow Curvature Method provides a better of the approximation of the slow invariant manifold of such system and, on the other hand, that it could also be used to highlight the bifurcation leading to a canard solution as emphasized by Ginoux and Llibre [10] in the case of the Van der Pol model.

The Jacobian matrix of system (33) evaluated at the pseudo singular points (35b) reads:

$$
J_{\left(F_{1}, G_{1}, G_{2}\right)}=\left(\begin{array}{ccc}
0 & 0 & -2(b-d)\left[s\left(\tilde{y}_{2}-\alpha\right)-\tilde{x}_{1}\right] \\
-2 d \tilde{y}_{2} & 0 & 2 d\left[s\left(\alpha-2 \tilde{y}_{2}\right)+\tilde{x}_{1}\right] \\
-1 & 0 & s
\end{array}\right) .
$$

According to Eqs. (26) we find that:

$$
\begin{aligned}
& p=\sigma_{1}=\operatorname{Tr}[J]=s, \\
& q=\sigma_{2}=-2(b-d)\left[s\left(\tilde{y}_{2}-\alpha\right)-\tilde{x}_{1}\right] .
\end{aligned}
$$


Thus, according to Prop. 1, the pseudo singular point is of saddle-type if and only if:

$$
-2(b-d)[s(\tilde{z}-\alpha)-\tilde{x}]<0 .
$$

In her work Shchepakina [20] used the following parameter set: $a=1, b=3$, $c=1, d=0.275255, I=2.7$ and $\alpha=-1.2$. According to Eq. (39) and with such a parameter set, i.e. $b-d>0$, the pseudo singular point is of saddle-type if and only if:

$$
s<\frac{4(b-d)^{3}+27 a^{2}(c+I)}{9 a[2(b-d)-3 a \alpha]}
$$

With $c=1, I=2.7$ and $\alpha=-1.2$, we find that: $s<2.2200954$. Thus, we have highlighted a canard with head in the Hindmarsh-Rose model (see Fig. 3) for the "duck parameter" value $s=2.220095<2.2200954$. For this parameters set the second-order approximation in $\varepsilon$ of the slow invariant manifold of the HindmarshRose model (28) can be provided while using the Flow Curvature Method introduced by Ginoux and Rossetto [9]. The result is presented in Fig. 3.

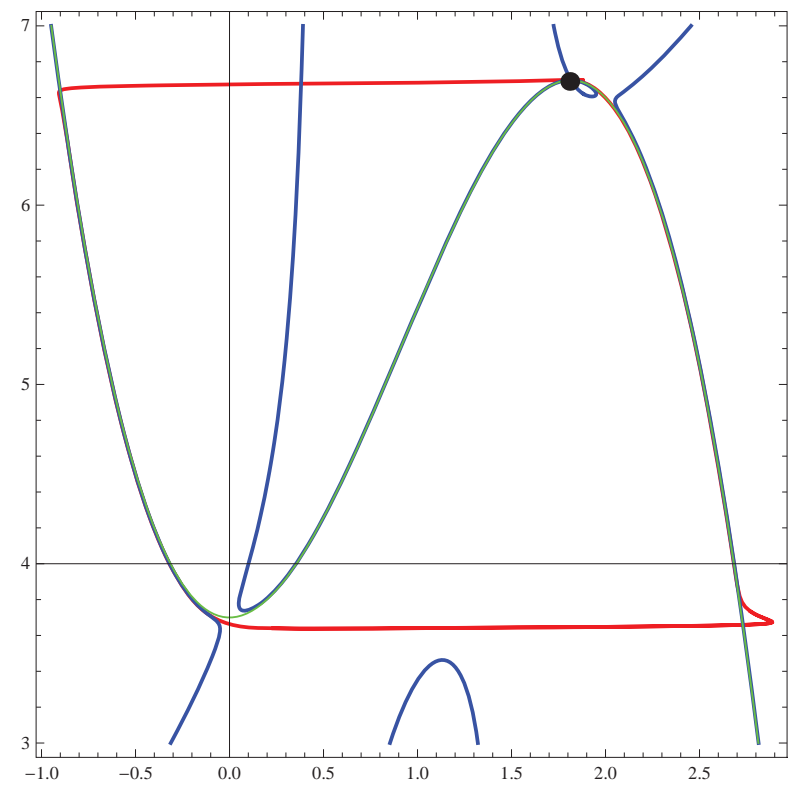

Figure 3. Canard solution of the Hindmarsh-Rose (28) model in the $(x, z)$ plane phase, its critical manifold (in green) and the second-order approximation in $\varepsilon$ of the slow invariant manifold (in blue) with the following parameter set: $a=1, b=3, c=1$, $d=0.275255, I=2.7, \alpha=-1.2$ and for the "duck parameter" value $s=2.220095$. 


\section{Discussion}

In a previous paper entitled: "Canards Existence in Memristor's Circuits" (see Ginoux \& Llibre [11]) we have proposed a new method for proving the existence of "canard solutions" for three and four-dimensional singularly perturbed systems with only one fast variable which is based on the stability of folded singularities of the normalized slow dynamics deduced from a well-known property of linear algebra. Thus, we proved that this unique condition is completely identical to that provided by Benoît [3], Szmolyan and Wechselberger [21] and Wechselberger [27]. In a second paper entitled: "Canards Existence in FitzHugh-Nagumo and Hodgkin-Huxley Neuronal Models" (see Ginoux \& Llibre [12]) we extended this method to the case of four-dimensional singularly perturbed systems with $k=2$ slow and $m=2$ fast variables. In this work we have extended this new method to the case of three-dimensional singularly perturbed systems with one slow and two fast variables and we have stated that the condition for the existence of "canard solutions" in such systems is exactly identical to those proposed in our previous paper. This result confirms the genericity of the condition $\left(\sigma_{2}<0\right)$ that we have highlighted and provides a simple and efficient tool for testing the occurrence of "canard solutions" in any three or four-dimensional singularly perturbed systems with one or two fast variables. Applications of this method to the famous coupled Hindmarsh-Rose model has enabled to confirm the existence of "canard solutions" in such systems as already stated by Shchepakina [20]. However, in this paper, only the case of pseudo singular points or folded singularities of saddle-type has been analyzed. Of course, the case of of pseudo singular points or folded singularities of node-type and focus-type could be also studied with the same method.

\section{ACKNOWLEDGMENTS}

The second author is partially supported by a FEDER-MINECO grant MTM201677278-P, a MINECO grant MTM2013-40998-P. The first author would like to thank Pr. J. Llibre for is kind invitation at Universitat Autònoma de Barcelona.

\section{REFERENCES}

[1] J. Argémi, Approche qualitative d'un problème de perturbations singulières dans $\mathbb{R}^{4}$, in Equadiff 1978, ed. R. Conti, G. Sestini, G. Villari (1978), 330-340.

[2] E. Benoît and C. Lobry, Les canards de $\mathbb{R}^{3}$, CR. Acad. Sc. Paris 294, Série I (1982) 483-488.

[3] E. Benoît, Systèmes lents-rapides dans $\mathbb{R}^{3}$ et leurs canards, Société Mathématique de France, Astérisque, (190-110) (1983) 159-191.

[4] N. Fenichel, Persistence and smoothness of invariant manifolds for flows, Ind. Univ. Math. J., 21 (1971) 193-225.

[5] N. Fenichel, Asymptotic stability with rate conditions, Ind. Univ. Math. J., 23 (1974) 11091137.

[6] N. Fenichel, Asymptotic stability with rate conditions II, Ind. Univ. Math. J., 26 (1977) 81-93.

[7] N. Fenichel, Geometric singular perturbation theory for ordinary differential equations, J. Diff. Eq. (1979) 53-98.

[8] R. FitzHugh, Impulses and physiological states in theoretical models of nerve membranes, Biophys. J., 1 (1961) 445-466.

[9] J.M. Ginoux and B. Rossetto, Slow Manifold of a Neuronal Bursting Model, in Emergent Properties in Natural and Artificial Dynamical Systems, Understanding Complex Systems, Series: Springer-Verlag, Heidelberg, Heidelberg, ed. M.A. Aziz-Alaoui and C. Bertelle, (2006), 119-128. 
[10] J.M. Ginoux and J. Llibre, Flow curvature method applied to canard explosion, Journal of Physics A: Mathematical and Theoretical, 44(46), 465203 (2011).

[11] J.M. Ginoux and J. Llibre, Canards in Memristor's Circuits, Qualitative Theory of Dynamical Systems, September 2015, 1-49.

[12] J.M. Ginoux and J. Llibre, Canards Existence in FitzHugh-Nagumo and Hodgkin-Huxley Neuronal Models, Mathematical Problems in Engineering, Vol. 15, Article ID 342010, 17 pages, 2015.

[13] J. Guckenheimer and R. Haiduc, Canards at folded nodes, Mosc. Math. J., 5(1) (2005) 91-103.

[14] J.L. Hindmarsh and R.M. Rose, A model of neuronal bursting using three coupled first order differential equations, Proceedings of the Royal Society of London. Series B, Biological Sciences 221 (1984), 87102. 246 (2009), 541-551.

[15] A.L. Hodgkin and A.F. Huxley, A quantitative description of membrane current and its application to conduction and excitation in nerve, J. Physiol. London 117 (1952), 500-544.

[16] C.K.R.T. Jones, Geometric Singular Perturbation Theory in Dynamical Systems, Montecatini Terme, L. Arnold, Lecture Notes in Mathematics, vol. 1609, Springer-Verlag (1994) $44-118$.

[17] T. Kaper, An Introduction to Geometric Methods and Dynamical Systems Theory for Singular Perturbation Problems, in Analyzing multiscale phenomena using singular perturbation methods, Baltimore, MD, (1998) 85-131. Amer. Math. Soc., Providence, RI.

[18] J.S. Nagumo, S. Arimoto and S. Oshizawa, An active pulse transmission line simulating nerve axon,Proc. Inst. Radio Engineers, 50 (1962) 2061-2070.

[19] A. Shilnikov and M. Kolomiets, Methods of the Qualitiative Theory for the Hindmarsh-Rose Model: A case study. A tutorial, International Journal of Bifurcation and Chaos, Vol. 18, No. 8 (2008) 2141-2168.

[20] , E. A. Shchepakina, Three scenarios for changing of stability in the dynamic model of nerve conduction, Mathematical Modelling. Information Technology and Nanotechnology (ITNT2016), Vol. 1638 (216) 664-673.

[21] P. Szmolyan and M. Wechselberger, Canards in $\mathbb{R}^{3}$, J. Dif. Eqs., 177 (2001) 419-453.

[22] F. Takens, Constrained equations, a study of implicit differential equations and their discontinuous solutions, in Structural stability, the theory of catastrophes and applications in the sciences, Springer Lecture Notes in Math., 525 (1976) 143-234.

[23] A.N. Tikhonov, On the dependence of solutions of differential equations on a small parameter, Mat. Sbornik N.S., 31 (1948) 575-586.

[24] K. Tchizawa, On relative stability in 4-dimensional duck solutions, Journal of Mathematics and System Sciences, 2(9) (2012) 558-563.

[25] K. Tchizawa, On the two methods for finding 4-dimensional duck solutions, Applied Mathematics, Scientific Research Publishing, 5(1) (2014) 16-24.

[26] M. Wechselberger, Existence and Bifurcation of Canards in $\mathbb{R}^{3}$ in the case of a Folded Node, SIAM J. Applied Dynamical Systems, 4 (2005) 101-139.

[27] M. Wechselberger, À propos de canards, Trans. Amer. Math. Soc., 364 (2012) 3289-3309.

1 Laboratoire LSIS, CNRS, UMR 7296, Université De Toulon, BP 20132, F-83957 LA Garde Cedex, France

E-mail address: ginoux@univ-tln.fr

2 Departament de Matemàtiques, Universitat Autònoma de Barcelona, 08193 Bellaterra, Barcelona, Catalonia, Spain

E-mail address: jllibre@mat.uab.cat

${ }^{3}$ Institute of Administration Engineering, Ltd., Tokyo, 101-0021, Japan

E-mail address: tchizawakiyoyuki@aim.com 